\title{
Under Nutrition and associated factors among lactating mothers in Southern Ethiopia: Institution Based Cross-sectional study
}

Gemechu Kejela ( $\square$ gemechukejela86@gmail.com )

Wollega University

Feleke Gebremeskel

Arba Minch University

Hadiya Hassen

Arba Minch University

Misgun Shewangizaw

Arba Minch University

Markos Desalegn

Jigjiga University

Research article

Keywords: Nutritional status, Arba Minch, Health center, lactating mother

Posted Date: December 17th, 2019

DOI: https://doi.org/10.21203/rs.2.18999/v1

License: (9) This work is licensed under a Creative Commons Attribution 4.0 International License.

Read Full License 


\section{Abstract}

Background Maternal Nutrition contributes significantly to the long term health of mothers and their children. Despite this importance, globally, about 795 million people are undernourished and women and their children are the most vulnerable groups.Objective the main aim of this study was to assess under nutrition and associated factors among lactating mothers in Arba Minch zuria district, Southern Ethiopia.

Methods institution based cross-sectional study was used. Data was collected using interview administered questionnaire from a total of 441 lactating mothers. To get the required respondents, first health centers in Arba Minch zuria district were selected by using lottery method and probability to population size was performed for each health center. Finally, eligible respondents were selected using systematic sampling method. The questionnaire was pretested and close supervision was undertaken during data collection to assure the quality of data. The collected data was entered using Epi data version 3.02 and exported to SPSS version 20.0 for analysis. Binary and multivariable logistic regression were conducted, and finally variables with $p$-value of $<0.05$ were considered as statistically significant predictors.

Results Prevalence of under nutrition in this study was $26.1 \%$. Under nutrition is higher among mothers who are unable to read and write $(\mathrm{AOR}[95 \% \mathrm{Cl}]=3.931[1.700,9.091])$, mothers who experienced more than five numbers of pregnancies $(A O R[95 \% \mathrm{Cl}]=2.453[1.051,5.728])$, those with more than four members of family size $(\mathrm{AOR}[95 \% \mathrm{Cl}]=2.289[1.171,4.472])$, those who live in the household with no toilet (AOR $[95 \% \mathrm{Cl}]=6.407[3.556,11.545])$ and those who have less than 4 antenatal care visits $(\mathrm{AOR}[95 \% \mathrm{Cl}]=$ $2.053[1.185,3.559])$.

Conclusions and Recommendations In this study, the magnitude of under nutrition among lactating women was high. So, nutrition based health information, education and communication is needed for mothers especially during pregnancy and lactation in the study area.

\section{Background}

Nutrition is a fundamental pillar of human life, health and development (1). Maternal nutrition during pregnancy and lactation influences the growth and development of the fetus. As a result, adequate nutrition for the mothers during lactation is important for the health of mothers and their children (2). Lactating mothers from low-income countries are nutritionally vulnerable group. The maternal nutrition requirement varies with respect to age, income and physiological changes like pregnancy and lactation $(1,3)$.

Inadequate quality and quantity diet is one of the major reasons for high levels of malnutrition in pregnant and lactating women (4). Due to the nursing process, mothers are subjected to nutritional stresses and pregnancy followed by lactation increase the health risk of mothers resulting in a high maternal mortality (3). Lack of sufficient calories, of macro- and micronutrients by the mothers during 
critical times like pregnancy and lactation can lead to deficiencies in building materials for the development and growth of the new born (5).

Household food insecurity, hunger and under-nutrition remain critical issues in different developing countries including Ethiopia (6). In Ethiopia, although there are maternal nutrition interventions that are efficacious and effective in improving maternal, neonatal, and child health (MNCH) outcomes, implementations have been limited $(7,8)$.

Malnutrition has been identified as a key underlying cause for maternal deaths. It pre-disposes pregnant and lactating women to an increased risk of infection, anemia, visual impairment, goiter among others. Environmental and economic conditions have huge impacts on the nutritional status of women in SubSaharan Africa. In this population, poverty limits food choices, thus affecting their quality of diet (9).

Ethiopia has reached Millennium Development Goal (MDG) 1, halving the number of undernourished people (from 75 percent to 35 percent over two decades). Despite these positive advances, Ethiopia remains one of the world's most food-insecure countries, where approximately one in three people live below the poverty line and about $27 \%$ of women age $15-49$ were undernourished (BMI of less than $18.5 \mathrm{~kg} / \mathrm{m} 2)(10)$.

To solve the problem of maternal malnutrition, micronutrient supplementation and food-based strategies such as diet diversity and food fortification have been reported in many studies in Sub-Saharan Africa (9, 11). Despite these efforts, the proportion of children and mothers affected by malnutrition is still high (2, $3,12)$. In addition, few studies in Ethiopia highlighted the issue of nutritional status among lactating mothers. So, the main aim of this study was to assess under nutrition and associated factors of lactating mothers in Arba Minch zuria district, Southern Ethiopia. The findings of this study is vital for policy makers, health care providers and any concerned bodies to design appropriate intervention strategies to tackle the problem.

\section{Methods And Materials}

Quantitative institution based cross-sectional study was conducted on lactating women who visited Arba Minch Zuria district health centers from June 5-20/2018. All lactating mothers visited public health centers of the area were the source population and all lactating mothers visited selected public health centers of the area during data collection period were the study population. Lactating mothers who lived in the area for more than six month and visited the public health centers of the area were included into the study. Lactating mothers who were critically ill, have hearing impairment and physical deformity that can alter anthropometric measurements were excluded from the study.

\subsection{Sample size determination and sampling procedures}

Sample size was calculated using single population proportion formula by considering the following assumptions: $\mathrm{P}=20 \%$ (prevalence of underweight among lactating mothers from the study conducted in 
Nekemte town (2), Significance level of $5 \%(a=0.05)$, and $Z a / 2=1.96$, Margin of error of $4 \%(d=0.04)$ and $10 \%$ non-response rate, the final sample size becomes 441 lactating mothers.

To get the eligible respondents, first, out of the seven health centers in Arba Minch zuria district, four of them were selected by using lottery method. Then, the calculated sample size was proportionally allocated to the health centers based on their population size according to the average number of clients registered prior to the study period in the respective health center. Finally the eligible respondents were selected by using systematic sampling method.

\subsection{Measurements}

In this study, lactating mother is a mother who is feeding breast milk for her infant/child during the study period. Body mass index is defined as the weight in kilograms divided by the square of the height in meters $\left(\mathrm{kg} / \mathrm{m}^{2}\right)$. Under-nutrition is defined as, lactating mothers with $\mathrm{BMI}$ of $\leq 18.5 \mathrm{~kg} / \mathrm{m}^{2}$. Individual Dietary Diversity Score is defined as, the sum of food groups eaten in a specified reference period.

Food secure households are those who were not experienced none of the food insecurity (access) conditions or just experienced worry, but rarely in the past 4 weeks. Food insecure households is inability of households to access sufficient food at all time to lead active and healthy life (includes all stages of food insecurity; mild, moderate and severe). Mildly food insecure households are households that worried about not having enough food sometimes or often, and/or are unable to eat preferred foods, and/or eat a more monotonous diet than desired and/or some foods considered undesirable, but only rarely. Moderately food insecure households are households sacrifices quality more frequently, by eating a monotonous diet or undesirable foods sometimes or often, and/or have started to cut back on quantity by reducing the size of meals or number of meals, rarely or sometimes. But it does not experience any of the three most severe conditions. Severely food insecure households are those household who has graduated to cutting back on meal size or number of meals often, and/or experienced any of the three most severe conditions (running out of food, going to bed hungry, or going a whole day and night without eating), even as infrequently as rarely.

\subsection{Data collection procedure}

Data was collected using structured interview administered questionnaire adopted from similar studies and modified based on the study variables and local context. The questionnaire first prepared in English and translated to Amharic and then translated back to English by bilingual expert, to check its Consistency. Twelve female diploma nurse data collectors and three MPH supervisors were recruited and trained for data collection.

\subsection{Data Quality Assurance}

To ensure the quality of data, training was given for data collectors and supervisors. Pre-test was conducted on $5 \%$ of the total sample to assess its clarity, length, completeness and consistency. The questionnaire was also translated to Amharic and close supervision was undertaken. 


\subsection{Data Processing and analysis}

Data was coded, entered into Epi data version 3.02 and exported to SPSS version 20.0 for analysis. Descriptive statistics was computed to determine the frequency and percentages. Binary logistic regression was conducted and $\mathrm{COR}$ with $95 \% \mathrm{Cl}$ was estimated to select the candidate variables for the final model. Then, variables with $p$-value of $<0.25$ at binary logistic regression were taken into multivariable logistic regression to control confounding. Hosmer-Lemeshow goodness-of-fit with stepwise (backward elimination) logistic regression was used to test for model fitness. AOR with $95 \% \mathrm{Cl}$ was estimated to assess the presence of association at multivariable logistic regression. Finally, variables with $p$-value of $<0.05$ were considered as statistically significant predictors of the outcome variable.

\section{Results}

\subsection{Socio-demographic characteristics of the study respondents}

Out of the total 445 sample size calculated for the study, 441 respondents responded making a response rate of $99.1 \%$. The age of the respondents ranges from 15 to 46 with mean and standard deviation of $26.98 \pm 5.67$. About $211(47.8 \%)$ respondents were in the age group of 17-25 years, 291 (66\%) were protestant and 438 (99.3\%) were married. About 124 (28.1\%) of mothers and $159(36.1 \%)$ of husbands were unable to read and write. Regarding their family size, $224(50.8 \%)$ of the respondents have 4-6 family members (Table 1 ). 
Table 1

Socio-demographic and economic characteristics of lactating mothers $(n=441)$ in Arba Minch zuria district, Southern Ethiopia, 2018.

\begin{tabular}{|llll|}
\hline Variables & Category & Frequency & Percentage \\
\hline Age of mothers (in year) & $17-25$ & 211 & 47.8 \\
& $26-35$ & 183 & 41.5 \\
& $36-49$ & 47 & 10.7 \\
\hline Religion & Orthodox & 150 & 34.0 \\
& Protestant & 291 & 66.0 \\
\hline Ethnicity & Gamo & 302 & 68.5 \\
& Gofa & 13 & 2.9 \\
& Wolayita & 24 & 5.4 \\
& Amhara & 13 & 2.9 \\
& Others* & 89 & 20.2 \\
\hline Maternal educational level & Unable to read and write & 124 & 28.1 \\
& Able to read and write & 92 & 20.9 \\
& primary & 136 & 30.8 \\
& secondary and above & 89 & 20.2 \\
\hline Husband's educational level & Unable to read and write & 159 & 36.1 \\
& read and write & 89 & 20.2 \\
& primary & 102 & 23.1 \\
& secondary and above & 91 & 20.6 \\
\hline Mother's Occupation & Farmer & 305 & 69.2 \\
& Merchant & 72 & 16.3 \\
& Government employer & 64 & 14.5 \\
\hline Family size & 1-3 members & 121 & 27.4 \\
& 4-6 members & 224 & 50.8 \\
\hline * Oyda, Basketo, Oromo & >6 members & 96 & 21.8 \\
\hline Naternal & & & \\
\hline
\end{tabular}

\subsection{Maternal Health Care and Feeding Practices}

Among the total respondents, 228 (51.7\%) were experienced one to two pregnancies. Majority of the respondents, $198(44.9 \%)$ spaced their children from $1-2$ years and $274(62.1 \%)$ had $\geq 4$ ANC visits during their last pregnancy. Majority of lactating mothers, 276 (62.6\%) were using family planning methods and out of these, $146(33.1 \%)$ uses injectable, whereas only $4(0.9 \%)$ uses pills. About 95 (21.5\%) of respondents had experienced diarrhea in the last two weeks preceding the study period. Majority of the respondents, $310(70.3 \%)$ has their own latrine. The mean \pm SD of lactating mothers dietary diversity score was $5.0 \pm 1.89$ and majority of them, $296(67.1 \%)$ had mean DDS of $\geq 5$ (Table 2). 
Table 2

Maternal health care and feeding practice of the study participants $(n=441)$ in Arba Minch zuria district, Southern Ethiopia, 2018.

\begin{tabular}{|c|c|c|c|}
\hline Variables & Category & Frequency & Percentage \\
\hline Number of pregnancy & $\begin{array}{l}1-2 \\
3-4 \\
5-6 \\
>6\end{array}$ & $\begin{array}{l}228 \\
107 \\
62 \\
44\end{array}$ & $\begin{array}{l}51.7 \\
24.2 \\
14.1 \\
10\end{array}$ \\
\hline Age of breast feeding child & $\begin{array}{l}\text { Less than } 6 \text { months } \\
6 \text { to } 11 \text { months } \\
12 \text { to } 24 \text { months }\end{array}$ & $\begin{array}{l}99 \\
158 \\
184\end{array}$ & $\begin{array}{l}22.4 \\
35.9 \\
41.7\end{array}$ \\
\hline Space $b / n$ index child and the previous birth & $\begin{array}{l}\text { It is the first child } \\
1-2 \text { years } \\
>2 \text { years }\end{array}$ & $\begin{array}{l}101 \\
198 \\
142\end{array}$ & $\begin{array}{l}22.9 \\
44.9 \\
32.2\end{array}$ \\
\hline Antenatal care for the last pregnancy & $\begin{array}{l}\text { Yes } \\
\text { No }\end{array}$ & $\begin{array}{l}413 \\
28\end{array}$ & $\begin{array}{l}93.7 \\
6.3\end{array}$ \\
\hline Number of antenatal care $(n=413)$ & $\begin{array}{l}\text { First ANC } \\
\text { Second ANC } \\
\text { Third ANC } \\
\text { Fourth ANC and above }\end{array}$ & $\begin{array}{l}12 \\
38 \\
89 \\
274\end{array}$ & $\begin{array}{l}2.7 \\
8.6 \\
20.2 \\
62.1\end{array}$ \\
\hline Current use of family planning & $\begin{array}{l}\text { Yes } \\
\text { No }\end{array}$ & $\begin{array}{l}276 \\
165\end{array}$ & $\begin{array}{l}62.6 \\
37.4\end{array}$ \\
\hline Which method is used $(n=276)$ & $\begin{array}{l}\text { Pills } \\
\text { Dipo } \\
\text { implant } \\
\text { IUCD }\end{array}$ & $\begin{array}{l}4 \\
146 \\
109 \\
17\end{array}$ & $\begin{array}{l}0.9 \\
33.1 \\
24.7 \\
3.9\end{array}$ \\
\hline The last time menstruation is seen & $\begin{array}{l}\text { In this month } \\
\text { Before one month } \\
\text { Don't know }\end{array}$ & $\begin{array}{l}118 \\
131 \\
192\end{array}$ & $\begin{array}{l}26.8 \\
29.7 \\
43.5\end{array}$ \\
\hline Time to breast feed & $\begin{array}{l}<6 \text { month } \\
6-11 \text { month } \\
12-24 \text { month } \\
>24 \text { month } \\
\text { do not know }\end{array}$ & $\begin{array}{l}21 \\
75 \\
260 \\
62 \\
23\end{array}$ & $\begin{array}{l}4.8 \\
17.0 \\
59.0 \\
14.0 \\
5.2\end{array}$ \\
\hline Start of complementary feeding & $\begin{array}{l}\text { Yes } \\
\text { No }\end{array}$ & $\begin{array}{l}345 \\
96\end{array}$ & $\begin{array}{l}78.2 \\
21.8\end{array}$ \\
\hline Age at start of complementary feeding & $\begin{array}{l}<6 \text { month } \\
\text { at } 6 \text { month } \\
>6 \text { month }\end{array}$ & $\begin{array}{l}80 \\
215 \\
50\end{array}$ & $\begin{array}{l}18.1 \\
48.8 \\
11.3\end{array}$ \\
\hline Mothers diarrhea in the last two weeks & $\begin{array}{l}\text { Yes } \\
\text { No }\end{array}$ & $\begin{array}{l}95 \\
346\end{array}$ & $\begin{array}{l}21.5 \\
78.5\end{array}$ \\
\hline Presence of toilet & $\begin{array}{l}\text { Yes } \\
\text { No }\end{array}$ & $\begin{array}{l}310 \\
131\end{array}$ & $\begin{array}{l}70.3 \\
29.7\end{array}$ \\
\hline
\end{tabular}




\begin{tabular}{|llll|}
\hline Variables & Category & Frequency & Percentage \\
\hline Source of drinking water & Tap & 279 & 63.3 \\
& Protected & 80 & 18.1 \\
& river/not protected & 66 & 15 \\
& Hole & 16 & 3.6 \\
\hline Nutrition education & Yes & 296 & 67.1 \\
& No & 145 & 32.9 \\
\hline Sources of nutrition education $(\mathrm{n}=296)$ & Health professionals & 251 & 56.9 \\
& Mass media & 45 & 10.2 \\
\hline Women dietary diversity score (WDDS) & $<5$ & 145 & 32.9 \\
& $\geq 5$ & 296 & 67.1 \\
\hline
\end{tabular}

\subsection{Household food security level of the study participants}

Household food security of the study respondents was assessed and classified as; food secured, mildly food in secured, moderately food in secured and severely food in secured. Accordingly, majority, 377 $(85.5 \%)$ of households were food secured and only $11(2.5 \%)$ of the respondents household were severely food in secured (Fig. 1).

\subsection{Under nutrition among lactating mothers}

The overall prevalence of under nutrition $(\mathrm{BMI}<18.5 \mathrm{~kg} / \mathrm{m} 2)$ among lactating mothers in this study was $26.1 \%$ (Fig. 2).

\subsection{Factors associated with under nutrition among lactating mothers}

\subsubsection{Binary Logistic Regression analysis}

In binary logistic regression analysis, maternal education (COR $[95 \% \mathrm{Cl}]=4.478[2.164,9.269])$, husband education $(\mathrm{COR}[95 \% \mathrm{Cl}]=4.65[2.237,9.672])$, having experienced $>3$ pregnancies $(\mathrm{COR}[95 \% \mathrm{Cl}]=$ $2.704[1.582,4.623]$, having $>4$ family size $(\mathrm{COR}[95 \% \mathrm{Cl}]=2.473[1.461,4.186])$, absence of toilet $(\mathrm{COR}[95 \% \mathrm{Cl}]=5.121[3.242,8.089]),<4$ ANC visits for the last pregnancy $(\mathrm{COR}[95 \% \mathrm{Cl}]=2.99[1.965,4.692])$, presence of maternal diarrhea $(\mathrm{COR}[95 \% \mathrm{Cl}]=1.824[1.120,2.970])$, absence of nutritional education $(\operatorname{COR}[95 \% \mathrm{Cl}]=1.870[1.205,2.902])$ and being severely food insecure $(\operatorname{COR}[95 \% \mathrm{Cl}]=5.176[1.483,18.073])$ shows significant association with under nutrition (Table 3 ). 
Table 3

Factors associated with under nutrition among lactating mothers in Arba Minch zuria district, Southern Ethiopia, 2018.

\begin{tabular}{|c|c|c|c|c|c|}
\hline \multirow[t]{2}{*}{ Variables } & \multirow[t]{2}{*}{ Category } & \multicolumn{2}{|c|}{$\begin{array}{l}\text { Under- } \\
\text { nutrition }\end{array}$} & \multirow[t]{2}{*}{$\operatorname{COR}[95 \% \mathrm{Cl}]$} & \multirow[t]{2}{*}{$\begin{array}{l}\text { P- } \\
\text { Value }\end{array}$} \\
\hline & & Yes & No & & \\
\hline Maternal age (in years) & $\begin{array}{l}17-25 \\
26-35 \\
36-49\end{array}$ & $\begin{array}{l}52 \\
48 \\
15\end{array}$ & $\begin{array}{l}159 \\
135 \\
32\end{array}$ & $\begin{array}{l}1 \\
1.087[0.690,1.713] \\
1.433[0.720,2.854]\end{array}$ & $\begin{array}{l}1 \\
0.718 \\
0.306\end{array}$ \\
\hline Maternal educational level & $\begin{array}{l}\text { Unable to read } \\
\text { and write } \\
\text { Able to read and } \\
\text { write } \\
\text { Primary } \\
\text { Secondary and } \\
\text { above }\end{array}$ & $\begin{array}{l}48 \\
39 \\
17 \\
11\end{array}$ & $\begin{array}{l}76 \\
53 \\
119 \\
78\end{array}$ & $\begin{array}{l}4.478[2.164,9.269] \\
5.218[2.453,11.097] \\
1.103[0.450,2.278] \\
1\end{array}$ & $\begin{array}{l}0.001 \\
0.001 \\
0.975 \\
1\end{array}$ \\
\hline Husband's educational level & $\begin{array}{l}\text { Unable to read } \\
\text { and write } \\
\text { Able to read and } \\
\text { write } \\
\text { Primary } \\
\text { Secondary and } \\
\text { above }\end{array}$ & $\begin{array}{l}58 \\
25 \\
22 \\
10\end{array}$ & $\begin{array}{l}101 \\
64 \\
80 \\
81\end{array}$ & $\begin{array}{l}4.651[2.237,9.672] \\
3.164[1.417,7.065] \\
2.227[0.992,5.001] \\
1\end{array}$ & $\begin{array}{l}0.001 \\
0.005 \\
0.052 \\
1\end{array}$ \\
\hline Mather's occupation & $\begin{array}{l}\text { Farmer } \\
\text { Merchant } \\
\text { G/ employee }\end{array}$ & $\begin{array}{l}77 \\
23 \\
15\end{array}$ & $\begin{array}{l}217 \\
58 \\
51\end{array}$ & $\begin{array}{l}1.236[0.658,2.322] \\
1.375[0.649,2.912] \\
1\end{array}$ & $\begin{array}{l}0.511 \\
0.406 \\
1\end{array}$ \\
\hline Number of pregnancy & $\begin{array}{l}\text { 1-2 pregnancies } \\
3-4 \text { pregnancies } \\
5-6 \text { pregnancies } \\
>6 \text { pregnancies }\end{array}$ & $\begin{array}{l}36 \\
36 \\
26 \\
17\end{array}$ & $\begin{array}{l}192 \\
71 \\
36 \\
27\end{array}$ & $\begin{array}{l}1 \\
2.704[1.582,4.623] \\
3,852[2.077,7.142] \\
3.358[1.662,6.786]\end{array}$ & $\begin{array}{l}1 \\
0.001 \\
0.001 \\
0.001\end{array}$ \\
\hline Family size & $\begin{array}{l}1-3 \text { members } \\
4-6 \text { members } \\
>6 \text { members }\end{array}$ & $\begin{array}{l}25 \\
65 \\
23\end{array}$ & $\begin{array}{l}117 \\
123 \\
73\end{array}$ & $\begin{array}{l}1 \\
2.473[1.461,4.186] \\
1.360[0.732,2.530]\end{array}$ & $\begin{array}{l}1 \\
0.001 \\
0.331\end{array}$ \\
\hline Age of breast feeding child & $\begin{array}{l}<6 \text { month } \\
6-11 \text { month } \\
12-24 \text { month }\end{array}$ & $\begin{array}{l}25 \\
41 \\
49\end{array}$ & $\begin{array}{l}74 \\
117 \\
135\end{array}$ & $\begin{array}{l}1 \\
1.037[0.583,1.846] \\
1.074[0.614,1.879]\end{array}$ & $\begin{array}{l}1 \\
0.901 \\
0.801\end{array}$ \\
\hline Source of drinking water & $\begin{array}{l}\text { Tap water } \\
\text { Protected } \\
\text { River/not } \\
\text { protected } \\
\text { Hole }\end{array}$ & $\begin{array}{l}68 \\
24 \\
18 \\
5\end{array}$ & $\begin{array}{l}211 \\
56 \\
48 \\
11\end{array}$ & $\begin{array}{l}1 \\
1.33[0.767,2.307] \\
1.164[0.634,2.135] \\
1.410[0.473,4.203]\end{array}$ & $\begin{array}{l}1 \\
0.310 \\
0.625 \\
0.537\end{array}$ \\
\hline
\end{tabular}

Keys: $\mathrm{COR}=$ Crude Odds Ratio; $\mathrm{Cl}=$ Confidence Interval; $\mathrm{CF}=$ Complementary Food; WDD = Women Dietary Diversity. 


\begin{tabular}{|c|c|c|c|c|c|}
\hline \multirow[t]{2}{*}{ Variables } & \multirow[t]{2}{*}{ Category } & \multicolumn{2}{|c|}{$\begin{array}{l}\text { Under- } \\
\text { nutrition }\end{array}$} & \multirow[t]{2}{*}{$\mathrm{COR}[95 \% \mathrm{Cl}]$} & \multirow[t]{2}{*}{$\begin{array}{l}\text { P- } \\
\text { Value }\end{array}$} \\
\hline & & Yes & No & & \\
\hline Presence of toilet & $\begin{array}{l}\text { Yes } \\
\text { No }\end{array}$ & $\begin{array}{l}50 \\
65\end{array}$ & $\begin{array}{l}260 \\
66\end{array}$ & $\begin{array}{l}1 \\
5.121[3.242,8.089]\end{array}$ & $\begin{array}{l}1 \\
0.001\end{array}$ \\
\hline $\begin{array}{l}\text { Presence of PNC for the last } \\
\text { pregnancy }\end{array}$ & $\begin{array}{l}\text { Yes } \\
\text { No }\end{array}$ & $\begin{array}{l}80 \\
35\end{array}$ & $\begin{array}{l}241 \\
85\end{array}$ & $\begin{array}{l}1 \\
1.240[0.777,1.980]\end{array}$ & $\begin{array}{l}1 \\
0.367\end{array}$ \\
\hline Number of ANC visit & $\begin{array}{l}<4 \text { visits } \\
\geq \text { visits }\end{array}$ & $\begin{array}{l}64 \\
45\end{array}$ & $\begin{array}{l}98 \\
206\end{array}$ & $\begin{array}{l}2.99[1.965,4.692] \\
1\end{array}$ & $\begin{array}{l}0.001 \\
1\end{array}$ \\
\hline Current use of family planning & $\begin{array}{l}\text { Yes } \\
\text { No }\end{array}$ & $\begin{array}{l}70 \\
45\end{array}$ & $\begin{array}{l}206 \\
120\end{array}$ & $\begin{array}{l}1 \\
1.104[0.713,1.708]\end{array}$ & $\begin{array}{l}1 \\
0.658\end{array}$ \\
\hline $\begin{array}{l}\text { The last time menstruation have } \\
\text { seen }\end{array}$ & $\begin{array}{l}\text { In this month } \\
\text { Before one month } \\
\text { Don't know }\end{array}$ & $\begin{array}{l}24 \\
31 \\
60\end{array}$ & $\begin{array}{l}94 \\
100 \\
132\end{array}$ & $\begin{array}{l}1 \\
1.214[0.665,2.218] \\
1.780[1.035,3.062]\end{array}$ & $\begin{array}{l}1 \\
0.528 \\
0.037\end{array}$ \\
\hline $\begin{array}{l}\text { Space between index child and } \\
\text { the previous birth }\end{array}$ & $\begin{array}{l}\text { It is } 1 \text { st child } \\
1-2 \text { years } \\
>2 \text { years }\end{array}$ & $\begin{array}{l}26 \\
54 \\
35\end{array}$ & $\begin{array}{l}75 \\
144 \\
107\end{array}$ & $\begin{array}{l}1.060[0.589,1.906] \\
1.146[0.70,1.878] \\
1\end{array}$ & $\begin{array}{l}0.846 \\
0.587 \\
1\end{array}$ \\
\hline Time of breast feeding & $\begin{array}{l}<6 \text { month } \\
6-11 \text { month } \\
12-24 \text { month } \\
>24 \text { month } \\
\text { Do not know }\end{array}$ & $\begin{array}{l}5 \\
20 \\
63 \\
22 \\
5\end{array}$ & $\begin{array}{l}16 \\
55 \\
197 \\
40 \\
18\end{array}$ & $\begin{array}{l}1 \\
1.164[0.377,3.592] \\
1.023[0.360,2.905] \\
1.760[0.568,5.453] \\
0.889[0.217,3.643]\end{array}$ & $\begin{array}{l}1 \\
0.792 \\
0.965 \\
0.327 \\
0.870\end{array}$ \\
\hline Start of CF & $\begin{array}{l}\text { Yes } \\
\text { No }\end{array}$ & $\begin{array}{l}91 \\
24\end{array}$ & $\begin{array}{l}254 \\
72\end{array}$ & $\begin{array}{l}1.075[0.639,1.808] \\
1\end{array}$ & $\begin{array}{l}0.786 \\
1\end{array}$ \\
\hline Age at start of CF & $\begin{array}{l}<6 \text { month } \\
\text { At } 6 \text { month } \\
>6 \text { month }\end{array}$ & $\begin{array}{l}20 \\
54 \\
17\end{array}$ & $\begin{array}{l}60 \\
161 \\
33\end{array}$ & $\begin{array}{l}1 \\
1.006[0.556,1.820] \\
1.545[0.713,3.350]\end{array}$ & $\begin{array}{l}1 \\
0.984 \\
0.270\end{array}$ \\
\hline Maternal diarrheal disease & $\begin{array}{l}\text { Yes } \\
\text { No }\end{array}$ & $\begin{array}{l}34 \\
81\end{array}$ & $\begin{array}{l}61 \\
265\end{array}$ & $\begin{array}{l}1.824[1.120,2.970] \\
1\end{array}$ & $\begin{array}{l}0.016 \\
1\end{array}$ \\
\hline Nutritional education & $\begin{array}{l}\text { Yes } \\
\text { No }\end{array}$ & $\begin{array}{l}65 \\
50\end{array}$ & $\begin{array}{l}231 \\
95\end{array}$ & $\begin{array}{l}1 \\
1.870[1.205,2.902]\end{array}$ & $\begin{array}{l}1 \\
0.005\end{array}$ \\
\hline Sources of nutritional education & $\begin{array}{l}\text { Health } \\
\text { professionals } \\
\text { Mass media }\end{array}$ & $\begin{array}{l}55 \\
10\end{array}$ & $\begin{array}{l}196 \\
35\end{array}$ & $\begin{array}{l}1 \\
1.018[0.474,2.185]\end{array}$ & $\begin{array}{l}1 \\
0.963\end{array}$ \\
\hline WDD mean score & $\begin{array}{l}<5 \\
>=5\end{array}$ & $\begin{array}{l}46 \\
69\end{array}$ & $\begin{array}{l}99 \\
227\end{array}$ & $1.529[0.983,2.377]$ & $\begin{array}{l}0.060 \\
1\end{array}$ \\
\hline
\end{tabular}

Keys: $\mathrm{COR}=$ Crude Odds Ratio; $\mathrm{Cl}=$ Confidence Interval; $\mathrm{CF}=$ Complementary Food; $\mathrm{WDD}=$ Women Dietary Diversity. 


\begin{tabular}{|c|c|c|c|c|c|}
\hline \multirow[t]{2}{*}{ Variables } & \multirow[t]{2}{*}{ Category } & \multicolumn{2}{|c|}{$\begin{array}{l}\text { Under- } \\
\text { nutrition }\end{array}$} & \multirow[t]{2}{*}{$\mathrm{COR}[95 \% \mathrm{Cl}]$} & \multirow[t]{2}{*}{$\begin{array}{l}\text { P- } \\
\text { Value }\end{array}$} \\
\hline & & Yes & No & & \\
\hline Household food security & $\begin{array}{l}\text { Food secure } \\
\text { Mildly food } \\
\text { insecure } \\
\text { Moderately food } \\
\text { insecure } \\
\text { Severely food } \\
\text { insecure }\end{array}$ & $\begin{array}{l}95 \\
6 \\
7 \\
7\end{array}$ & $\begin{array}{l}282 \\
16 \\
24 \\
4\end{array}$ & $\begin{array}{l}1 \\
1.109[0.422,2.916] \\
0.863[0.360,2.066] \\
5.176[1.483, \\
18.073]\end{array}$ & $\begin{array}{l}1 \\
0.834 \\
0.740 \\
0.010\end{array}$ \\
\hline
\end{tabular}

\subsubsection{Multivariable Logistic Regression Analysis}

In multivariable logistic regression analysis, variables like maternal education, number of pregnancy, family size, presence of toilet and number of antenatal care visits shows significant association with under nutrition.

Mothers who are unable to read and write were almost four times more likely to be undernourished compared to those with secondary and above educational levels $(\mathrm{AOR}[95 \% \mathrm{Cl}]=3.93[1.700,9.091])$. Respondents who experienced 5-6 number of pregnancies were 2.45 times more likely to be undernourished compared to those who experienced 1-2 pregnancies $(\mathrm{AOR}[95 \% \mathrm{Cl}]=2.453[1.051$, 5.728]). Similarly, respondents who have family size of 4-6 members were 2.3 times more likely to be under nourished compared to those who have family size of $1-3$ members $(A O R[95 \% \mathrm{Cl}]=2.289[1.171$, 4.472]).

Respondents who live in the household with no toilet were 6.4 times more likely to be undernourished compared to their counterparts $(\mathrm{AOR}[95 \% \mathrm{Cl}]=6.407[3.556,11.545])$. Respondents who visited $<4$ ANC were 2 times more likely to be under nourished compared to those who visited greater than or equal to four $(\mathrm{AOR}[95 \% \mathrm{Cl}]=2.053[1.185,3.559])($ Table 4$)$. 
Table 4

Multivariable logistic regression showing factors associated with under nutrition among lactating mothers in Arba Minch zuria district, Southern Ethiopia, 2018

\begin{tabular}{|c|c|c|c|c|c|}
\hline \multirow[t]{2}{*}{ Variables } & \multirow[t]{2}{*}{ Category } & \multicolumn{2}{|c|}{$\begin{array}{l}\text { Under- } \\
\text { nutrition }\end{array}$} & \multirow[t]{2}{*}{$\mathrm{COR}[95 \% \mathrm{Cl}]$} & \multirow[t]{2}{*}{$\mathrm{AOR}[95 \% \mathrm{Cl}]$} \\
\hline & & Yes & No & & \\
\hline $\begin{array}{l}\text { Maternal educational } \\
\text { level }\end{array}$ & $\begin{array}{l}\text { Unable to read } \\
\text { and write } \\
\text { Able to read } \\
\text { and write } \\
\text { Primary } \\
\text { Secondary and } \\
\text { above }\end{array}$ & $\begin{array}{l}48 \\
39 \\
17 \\
11\end{array}$ & $\begin{array}{l}76 \\
53 \\
119 \\
78\end{array}$ & $\begin{array}{l}4.478[2.164,9.269] \\
5.218[2.453,11.097] \\
1.103[0.450,2.278] \\
1\end{array}$ & $\begin{array}{l}3.931[1.700,9.091]^{\star} \\
3.991[1.690,9.425]^{\star} \\
0.803[0.329,1.961] \\
1\end{array}$ \\
\hline $\begin{array}{l}\text { Husband's } \\
\text { educational level }\end{array}$ & $\begin{array}{l}\text { Unable to read } \\
\text { and write } \\
\text { Able to read } \\
\text { and write } \\
\text { Primary } \\
\text { Secondary and } \\
\text { above }\end{array}$ & $\begin{array}{l}58 \\
25 \\
22 \\
10\end{array}$ & $\begin{array}{l}101 \\
64 \\
80 \\
81\end{array}$ & $\begin{array}{l}4.651[2.237,9.672] \\
3.164[1.417,7.065] \\
2.227[0.992,5.001] \\
1\end{array}$ & $\begin{array}{l}2.403[0.964,5.986] \\
1.065[0.377,3.014] \\
1.791[0.674,4.757] \\
1\end{array}$ \\
\hline Number of pregnancy & $\begin{array}{l}1-2 \\
\text { pregnancies } \\
3-4 \\
\text { pregnancies } \\
5-6 \\
\text { pregnancies } \\
>6 \text { pregnancies }\end{array}$ & $\begin{array}{l}36 \\
36 \\
26 \\
17\end{array}$ & $\begin{array}{l}192 \\
71 \\
36 \\
27\end{array}$ & $\begin{array}{l}1 \\
2.704[1.582,4.623] \\
3,852[2.077,7.142] \\
3.358[1.662,6.786]\end{array}$ & $\begin{array}{l}1 \\
1.312[0.630,2.731] \\
2.453[1.051, \\
5.728]^{\star} \\
1.720[0.684,4.327]\end{array}$ \\
\hline Family size & $\begin{array}{l}1-3 \text { members } \\
4-6 \text { members } \\
>6 \text { members }\end{array}$ & $\begin{array}{l}25 \\
65 \\
23\end{array}$ & $\begin{array}{l}117 \\
123 \\
73\end{array}$ & $\begin{array}{l}1 \\
2.473[1.461,4.186] \\
1.360[0.732,2.530]\end{array}$ & $\begin{array}{l}1 \\
2.289[1.171, \\
4.472]^{\star} \\
0.786[0.366,1.687]\end{array}$ \\
\hline Presence of toilet & $\begin{array}{l}\text { Yes } \\
\text { No }\end{array}$ & $\begin{array}{l}50 \\
65\end{array}$ & $\begin{array}{l}260 \\
66\end{array}$ & $\begin{array}{l}1 \\
5.121[3.242,8.089]\end{array}$ & $\begin{array}{l}1 \\
6.407[3.556 \\
11.545]^{\star}\end{array}$ \\
\hline Number of ANC visit & $\begin{array}{l}<4 \text { visits } \\
\geq \text { visits }\end{array}$ & $\begin{array}{l}64 \\
45\end{array}$ & $\begin{array}{l}98 \\
206\end{array}$ & $\begin{array}{l}2.99[1.965,4.692] \\
1\end{array}$ & $\begin{array}{l}2.053[1.185 \\
3.559]^{\star} \\
1\end{array}$ \\
\hline $\begin{array}{l}\text { The last time } \\
\text { menstruation have } \\
\text { seen }\end{array}$ & $\begin{array}{l}\text { In this month } \\
\text { Before } 1 \text { month } \\
\text { Don't know }\end{array}$ & $\begin{array}{l}24 \\
31 \\
60\end{array}$ & $\begin{array}{l}94 \\
100 \\
132\end{array}$ & $\begin{array}{l}1 \\
1.214[0.665,2.218] \\
1.780[1.035,3.062]\end{array}$ & $\begin{array}{l}1 \\
0.864[0.394,1.893] \\
1.143[0.562,2.327]\end{array}$ \\
\hline
\end{tabular}

Keys: $\mathrm{COR}=$ Crude Odds ratio; $\mathrm{AOR}=$ Adjusted Odds Ratio; $\mathrm{Cl}=$ Confidence Interval; $\mathrm{WDD}=$ Women Dietary Diversity; * $=$ P-value $<0.05$

8.7. List of figures 


\begin{tabular}{|c|c|c|c|c|c|}
\hline \multirow[t]{2}{*}{ Variables } & \multirow[t]{2}{*}{ Category } & \multicolumn{2}{|c|}{$\begin{array}{l}\text { Under- } \\
\text { nutrition }\end{array}$} & \multirow[t]{2}{*}{ COR[95\%Cl] } & \multirow[t]{2}{*}{ AOR[95\%Cl] } \\
\hline & & Yes & No & & \\
\hline $\begin{array}{l}\text { Maternal diarrheal } \\
\text { disease }\end{array}$ & $\begin{array}{l}\text { Yes } \\
\text { No }\end{array}$ & $\begin{array}{l}34 \\
81\end{array}$ & $\begin{array}{l}61 \\
265\end{array}$ & $1.824[1.120,2.970]$ & $\begin{array}{l}1.786[0.953,3.347] \\
1\end{array}$ \\
\hline Nutritional education & $\begin{array}{l}\text { Yes } \\
\text { No }\end{array}$ & $\begin{array}{l}65 \\
50\end{array}$ & $\begin{array}{l}231 \\
95\end{array}$ & $\begin{array}{l}1 \\
1.870[1.205,2.902]\end{array}$ & $\begin{array}{l}1 \\
1.439[0.787,2.632]\end{array}$ \\
\hline WDD mean score & $\begin{array}{l}<5 \\
>=5\end{array}$ & $\begin{array}{l}46 \\
69\end{array}$ & $\begin{array}{l}99 \\
227\end{array}$ & $\begin{array}{l}1.529[0.983,2.377] \\
1\end{array}$ & $1.005[0.535,1.890]$ \\
\hline $\begin{array}{l}\text { Household food } \\
\text { security }\end{array}$ & $\begin{array}{l}\text { Food secure } \\
\text { Mildly food } \\
\text { insecure } \\
\text { Moderately } \\
\text { food insecure } \\
\text { Severely food } \\
\text { insecure }\end{array}$ & $\begin{array}{l}95 \\
6 \\
7 \\
7\end{array}$ & $\begin{array}{l}281 \\
16 \\
24 \\
4\end{array}$ & $\begin{array}{l}1 \\
1.109[0.422,2.916] \\
0.863[0.360,2.066] \\
5.176[1.483,18.073]\end{array}$ & $\begin{array}{l}1 \\
1.685[0.485,5.857] \\
0.570[0.159,2.045] \\
2.004[0.349, \\
11.505]\end{array}$ \\
\hline \multicolumn{6}{|c|}{$\begin{array}{l}\text { Keys: } \mathrm{COR}=\text { Crude Odds ratio; } \mathrm{AOR}=\text { Adjusted Odds Ratio; } \mathrm{Cl}=\text { Confidence Interval; } \mathrm{WDD}=\text { Women } \\
\text { Dietary Diversity; }{ }^{*}=\mathrm{P} \text {-value }<0.05\end{array}$} \\
\hline 8.7. List of figures & & & & & \\
\hline
\end{tabular}

\section{Discussion}

In this study, about $26.1 \%$ of lactating mothers were undernourished. Under nutrition was more common among mothers who are unable to read and write, those who experienced high number of pregnancies, those with high family size, those who reside in the household with no toilet, and those who have less than four ANC visits for their last pregnancy.

The prevalence of under nutrition in this study was comparable with findings of study conducted in Rayitu district of Ethiopia (24\%) (13), Alamata, Tigiray (24.6) (14) and Womberma district of North West Ethiopia (25.4\%) (15). However, the prevalence is lower than study conducted in Samre district of Tigray (31\%) (3), and Dedo and Seka chekors's of Jimma district (40.6\%) (16). The difference might be as a result of improvement in nutritional education, improvement in socio-economy and difference in geographical setting. But, the finding of this study is higher than study conducted in Tanzania (11\%) (17), Nigeria (5\%) (18), Nekemte town (20\%) (2), Ambo districts (21.5\%) (19), and Offa district of Wolayta zone $(15.8 \%)(20)$. The reason for this discrepancy may be due to difference in socio-economy, geographical settings, and seasonal variation.

Mothers who are unable to read and write were almost four times more likely to be under nourished compared to those who have secondary and above educational level. This finding is consistent with studies conducted in Bangladesh (21), Ambo district (19), Adama district (22), and Offa district (20). The 
possible reason may be since those who are able to read and write can get nutritional information through reading books, posters and magazines than those who are unable to read and write.

Respondents who experienced 5-6 pregnancies were 2.5 times more likely to be under nourished compared to those who experienced 1-2 pregnancies. The finding of this study was inconsistent with study conducted in Alamat highland of Tigray in which respondents who have higher pregnancies were $44 \%$ less likely to be undernourished compared to those who lower pregnancies (14). However, this finding is supported by study conducted in Babile, Ethiopia, which shows, as parity increased by one unit, the BMI of the lactating mothers decreased (23). The possible reason may be, because of the fact that, as the number of pregnancy increases, exposure to different health problems including under nutrition may occur. In addition, the mother herself may be biologically depleted from too frequent births, which could also negatively affect the nutritional status of herself and her newborn.

Respondents whose family size is greater than four members were 2.29 times more likely to be under nourished compared to those who have less family size. This finding is supported by the study conducted in Womberma district of North West Ethiopia (15) and Nekemte town (2). This may be due shortages of food in the household with high family size, and culturally mothers always eat least food (some time leftover) at the last which leads to under nutrition.

Respondents who have toilet were 6.4 times more likely to be undernourished compared to their counterparts. This is supported by study conducted in Adama district (22). The reason might be due to the fact that, open defecation, because of lack of toilet results in an increased risk of diarrheal disease, which might contributes to under nutrition. In addition, poor handling and disposal of household wastes including human excreta are major causes of environmental pollution, which creates breeding grounds for pathogenic microorganism that causes under nutrition.

Respondents who visited ANC $<4$ times were two times more likely to be undernourished compared to those who visited $\geq 4$ times. This finding is consistent with the study conducted in Samre district of Tigray (3) and Rayitu districts of Ethiopia (13). This may be because of the fact that, ANC time is a time when nutritional information and other support for healthy behaviors is widely provided and might contribute to the lower probability of getting under nutrition.

\section{Limitations}

- Since some question asks about past events, recall bias may occur

- An anthropometric measurement error may also occur

\section{Conclusions And Recommendations 6.1.Conclusions}


The result of this study shows slightly high proportion of under nutrition among lactating mothers.

Predictors of under nutrition among lactating mothers in this study includes: maternal educational level, number of pregnancy, family size, presence of toilet and number of ante natal care visits.

\subsection{Recommendations}

Based on the finding of this study, the following recommendations were made:

\section{Arba Minch woreda health office:}

- Should strengthen health education programs on proper maternal and child dietary practices and the need of adequate dietary intake during pregnancy and lactation in order to improve health and nutrition outcomes of lactating mothers and their children.

- Since health extension workers are more close to the community, woreda health office should work with health extension workers to increase awareness of lactating mothers on how to improve their own nutritional status.

- Woreda health office should have to work with different sectors like; educational and agricultural sectors to improve women educational level and then, their nutritional status.

\section{Health Extension workers:}

- Health extension workers should have to closely work with the community and provide health information like; importance of adequate nutrition during lactation, importance of having few children based on their capacity, importance of having toilet and proper use of it, and importance of antenatal and post natal visits for the community.

\section{Researchers:}

- Further research that uses advanced design is needed to identify more variables that determine the nutritional status of lactating mothers.

\section{Abbreviations}

ANC

AOR

$\mathrm{BMI}$

$\mathrm{Cl}$

COR
Antenatal Care

Adjusted Odds Ratio

Body Mass Index

Confidence Interval

Crude Odds Ratio 
EDHS Ethiopian Demographic and Health Survey

FAO Food and Agricultural Organization

MDG Millennium Development Goal

$\mathrm{MNCH} \quad$ Maternal, Neonatal and Child Health

SNNPR Southern Nation and Nationalities and Peoples Region

USAID United States Agency for International Development

\section{Declarations}

\subsection{Ethical approval and consent to participate}

Ethical clearance was obtained from Arba Minch University Research Ethic review committee to conduct the study. In addition permission was obtained from Gamo Gofa zone health Department, managers of health centers. Confidentiality was secured by informing and giving awareness to the data collectors not to record identifiers of the client and disseminate any information obtained from client.

\subsection{Consent for publication}

Not applicable

\subsection{Availability of data and materials}

The data collected for this study can be obtained from the corresponding author based on a reasonable request.

\subsection{Funding}

The data collection process of this study was funded by Arba Minch University for the support of data collection. The funding body only followed the process to check whether the fund allocated was used for the proposed research.

\subsection{Authors' Contribution}

GK wrote the proposal, participated in data collection, analyzed the data and drafted the paper. FG and $\mathrm{HH}$ approved the proposal with great revisions and revised subsequent drafts of the paper. MS and MD contributed in the designing of the methodology and write-up. All authors and read and approved the final manuscript. 


\subsection{Conflict of Interest}

The authors declare that they have no conflict of interest regarding the publication of the paper.

\subsection{Acknowledgment}

Authors would like to thank Arba Minch University, College of medicine and health sciences for initiating them to conduct this study and financial support. Deep appreciations have gone to managers of the health centers. Also, special thanks to data collectors, supervisors and all peoples who involved in the study directly or indirectly.

\section{References}

1. Daba G., Beyene F., Fekadu H., Garoma W., Assessment of Knowledge of Pregnant Mothers on Maternal Nutrition and Associated Factors in Guto Gida Woreda, East Wollega Zone, Ethiopia. J Nutrition Disorders 2013, 4:130.

2. Temesgen H., Habtamu G., Dessalegn W. Dunkana K., Nutritional Status and Associated Factors among Lactating Mothers in Nekemte Referral Hospital and Health Centers, Ethiopia. Journal of Food Science and Quality Management, 2015. Vol.35: 2224-6088.

3. Kiday H., Afework M. and Meron G. Feeding practices, nutritional status and associated factors of lactating women in Samre Woreda, South Eastern Zone of Tigray, Ethiopia, 2013. Nutrition Journal, 12:28 http://www.nutritionj.com/content/12/1/28

4. Desalegn K., Pragya S., Debebe M. and Tefera B. Nutritional Status and Associated Factors among Pregnant Women in Wondo Genet District, Southern Ethiopia, Journal of Food Science and Engineering, 2015. 5: 85-94.

5. Federico M., Sara B., Serena O., Giorgio M. Nutrition in pregnancy and lactation: how a healthy infant is born, Florence, Italy. Journal of Pediatric and Neonatal Individualized Medicine, 2015; 4(2): e040236.

6. USAID: Ethiopia: Nutrition Profile, Updated June, 2014.

7. Lisa S., Laura B., Jessica W., Afework M., Solomon M., Alemzewed R., Hiwot A. and John M. Policies and program implementation experience to improve maternal nutrition in Ethiopia: Food and Nutrition Bulletin, 2012. 33(2).

8. FAO, IFAD and WFP. The State of Food Insecurity in the World 2014. Strengthening the enabling environment for food security and nutrition. Rome.

9. O.B. Oluwole, A.A. Agboola, J. Onyibe, and O.A. Adeyoju. Improving Maternal Nutrition in Nigeria: $A$ Review, 2016. 1(2): 17-22. Available online at: http://www.prudentjournals.org/IRJAFS.

10. CSA (Central Statistical Agency). Ethiopia Demographic and Health Survey 2011: Maryland, USA: ICF International, 2012. Available at http://www.unicef.org/ethiopia. 
11. USAID: Maternal diet and nutrition practices and their determinants: A report on formative research findings and recommendations for social and behavior change communication programming in the Amhara, Oromia, SNNP and Tigray regions of Ethiopia, 2014.

12. K. L. Lindsay, E. R. Gibney \& F. M. McAuliffe. Maternal nutrition among women from Sub-Saharan Africa, with a focus on Nigeria, and potential implications for pregnancy outcomes among immigrant populations in developed countries: Journal of Human Nutrition and Dietetics, 2012.

13. Betemariam G., Sibhatu B., Zinaw T., Tsigereda L., Mekitew L. Determinants of malnutrition among pregnant and lactating women under humanitarian setting in Rayitu, Ethiopia. BMC Nutrition (2018) 4:11

14. Ismael S., Kiday H., Yohannes A. Comparison of nutritional status and associated factors of lactating women between lowland and highland communities of District Raya, Alamata, Southern Tigiray, Ethiopia. BMC Nutrition (2017) 3:61 DOI 10.1186/s40795-017-0179-6

15. Sileshi B., Getachew K., Muluken T. Factors associated with underweight among lactating women in Womberma woreda, Northwest Ethiopia; a cross-sectional study. BMC Nutrition (2017) 3:46 DOI 10.1186/s40795-017-0165-z

16. Mihiretu A., Alemayehu A., Abebe M. Factors Associated with Malnutrition among Lactating Women in Subsistence Farming Households from Dedo and Seqa-Chekorsa Districts, Jimma Zone. Developing Country Studies www.iiste.org, 2014. 5 (21).

17. AH Mtumwa. (2016) Determinants of under nutrition among women of reproductive age in Tanzania mainland, South African. Journal of Clinical Nutrition, 29:2, 75-81, DOI: 10.1080/16070658.2016.1216509

18. Sanusi A. and Falana A. The Nutritional Status of Mothers Practicing Breast Feeding In Ibadan, Nigeria. Afr. J. Biomed. Res. (2009) 12: 2.

19. Eshetu Z., Gudina E., Firhiwot M. Under nutrition and associated factors among lactating mothers in rural Ambo district, West Shewa Zone, Oromia region, Ethiopia. Advances in Nutrition, Food Science and Technology (2016) 1:

20. Berhanu J.; Abebe H.; Girma A.; Solomon E.; Desalegn K.; Tsehai A. (2018). Chronic Energy Deficiency and Associated Factors among Lactating Mothers (15-49 years old) in Offa Woreda, Wolayita Zone, SNNPRs, Ethiopia. World Scientific Research, 5(1): 13-23.

21. Rezwan M., Sharif E. and Tahomina J. Mothers' Breastfeeding Practices and Nutritional Status in the Slums of Bangladesh: A Study Based on Khulna City: International Journal of Multidisciplinary Research, 2015.2 (4):7-18.

22. Abeya SG, Biru KM, Jima A (2018) Factors Associated with Underweight among Lactating Mothers in Adama District, Oromia Region, Ethiopia. J Orthop Bone Res 1: 101

23. Kedir R., Thomas O., Tefera B., Nora B. Seasonal variation in nutritional status and anemia among lactating mothers in two agro-ecological zones of rural Ethiopia: A longitudinal study: Nutrition, 2015. 31:1213-1218. 


\section{Figures}

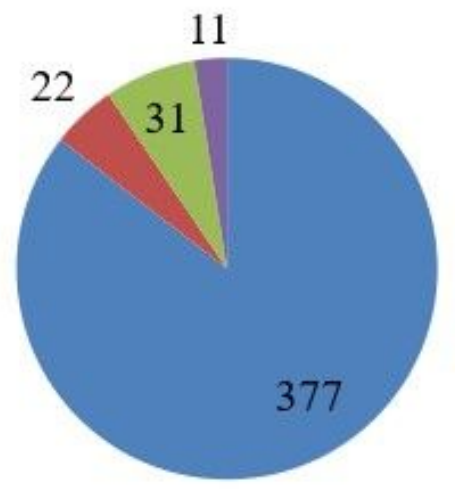

- Secured

Mildly insecured

Moderetly insecured

Severly insecured

\section{Figure 1}

Household food security levels among lactating mothers $(n=441)$ in Arba Minch zuria district, Southern Ethiopia, 2018

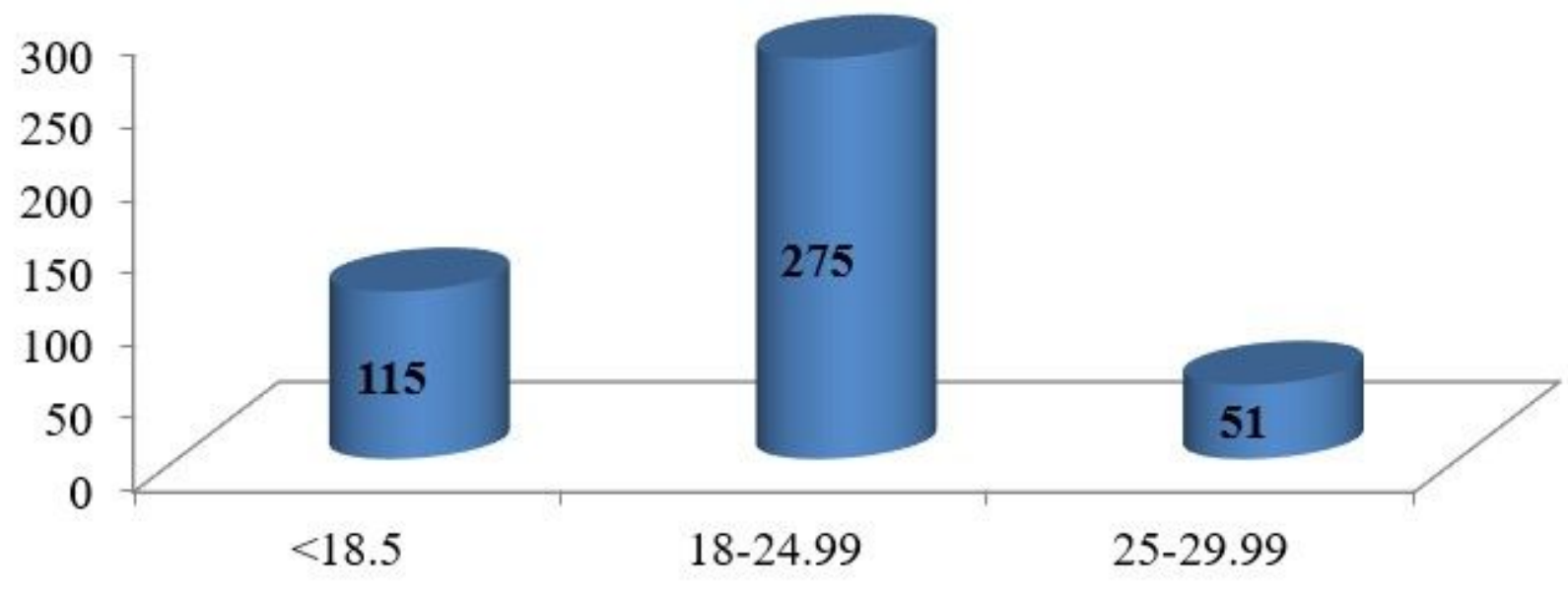

BMI category $(\mathrm{kg} / \mathrm{m} 2)$

\section{Figure 2}

Bar chart showing nutritional status of lactating mothers $(n=441)$ in Arba Minch zuria district, Southern Ethiopia, 2018. 